\title{
Static and Dynamic Light Scattering by Concentrated Colloidal Suspensions of Polydisperse Sterically Stabilized Boehmite Rods
}

\author{
Johan Buitenhuis, Jan K. G. Dhont, and Henk N. W. Lekkerkerker* \\ Van't Hoff Laboratory, University of Utrecht, Padualaan 8 , \\ $3584 \mathrm{CH}$ Utrecht, The Netherlands \\ Received May 9, 1994; Revised Manuscript Received August 19, 1994
}

\begin{abstract}
Static and dynamic light-scattering experiments have been performed on isotropic dispersions of sterically stabilized polydisperse boehmite rods, with an average length to width ratio of 6.4 . Measurements were made up to concentrations close to the isotropic nematic phase separation. At high concentrations, the scattered intensity autocorrelation function of the rods is found to separate in two modes that are attributed to collective and exchange diffusion. Corresponding mode amplitudes are in fair agreement with results of the polydisperse scaled particle theory. Whereas the collective mode decay time is hardly concentration dependent, the exchange mode decay time decreases exponentially with concentration
\end{abstract}

\section{Introduction}

Many experimental model studies on structural and dynamical properties of concentrated colloidal systems have been performed using static and dynamic light scattering. ${ }^{1-9}$ Most of these studies are devoted to spherical particles, although several experiments on rod-shaped virus particles (mostly tobacco mosaic virus $)^{7,8}$ and rigid rodlike polymers ${ }^{7,9}$ have been performed.

Here we present a static and dynamic light-scattering study on dispersions of poly(isobutene)-grafted boehmite rods, the synthesis of which was recently developed in our laboratory. ${ }^{10,11}$ The rods used in this study have an average length of $71 \mathrm{~nm}$ and an average width of $11.1 \mathrm{~nm}$, with a polydispersity of about $40 \%$. The concentration ranges from the dilute regime to the concentrated regime close to the isotropic nematic phase separation.

The main feature of the dynamic light-scattering (DLS) measurements is the separation of the autocorrelation functions at higher concentrations in a fast and slow mode. Analogous to a system of spherical particles, this is interpreted as being due to the polydispersity. Following this analogy, the fast mode is attributed to collective diffusion and the slow mode is attributed to exchange (or self-) diffusion..$^{2,12,13}$ The corresponding mode amplitudes, resulting from the combination of dynamic and static light-scattering (SLS) measurements, are compared to results from the scaled particle theory (SPT) for polydisperse rods, which recently has been extended to calculate the scattered intensity at zero scattering angle..$^{14}$

A complication affecting the measurements is a moderate degree of clustering in the dispersions, which, so far, we have not been able to avoid. Fortunately, this clustering gives rise to a well-separated additional decay mode in the autocorrelation function. This allows the separation of the scattering in a part due to clusters and in a part due to individual rods.

In section 2 we outline the static and dynamic lightscattering theory for polydisperse rod-shaped particles. Section 3 gives the experimental details, and in section 4 the characterization and dispersion properties are

\footnotetext{
* To whom correspondence should be addressed.

$\otimes$ Abstract published in Advance ACS Abstracts, October 15, 1994 .
}

$0024-9297 / 94 / 2227-7267 \$ 04.50 / 0$ described. The results obtained with static light scattering and the presence of clusters are discussed in section 5, whereas the dynamic light-scattering measurements are discussed in section 6 . Here section 6.1 describes the scattering of a dilute dispersion, and section 6.2 gives the scattering as a function of the concentration. Finally, some concluding remarks will be given in section 7 . In the Appendix the explicit equations are presented, describing the length and width polydispersities that are used in several model calculations.

\section{Light-Scattering Theory}

2.1. Preliminaries. We consider the scattering of an isotropic dispersion of $N$ polydisperse rods in a scattering volume, $V_{s}$. Within the Rayleigh-GansDebye (RGD) approximation, the time-averaged scattered intensity for vertically polarized incident (and scattered) light of intensity $I_{0}$ is given by ${ }^{2-5}$

$$
<I(k)>=N \overline{<b^{2}(\mathbf{k}, \hat{\mathbf{u}})>} S^{\mathrm{M}_{(k)}}
$$

Here $S^{\mathrm{M}}(k)$ is the "measured" structure factor describing the interparticle interference,

$$
\begin{aligned}
S^{\mathrm{M}_{(}}(k)=\left[N \overline{<b^{2}(\mathbf{k}, \hat{\mathbf{u}})>}\right]^{-1} \sum_{i} \sum_{j}<b_{i}\left(\mathbf{k}, \hat{\mathbf{u}}_{i}\right) b_{j}\left(\mathbf{k}, \hat{\mathbf{u}}_{j}\right) \times \\
\exp \left[i \mathbf{k} \cdot\left(\mathbf{r}_{i}-\mathbf{r}_{j}\right)\right]>
\end{aligned}
$$

with $\mathbf{r}_{i}$ and $\mathbf{r}_{j}$ the positions, $b_{i}$ and $b_{j}$ the scattering amplitudes, and $\hat{\mathbf{u}}_{i}$ and $\hat{\mathbf{u}}_{j}$ the orientation vectors of particle $i$ and $j$, respectively. The angular brackets denote ensemble averaging over all possible positions and orientations, whereas a bar indicates number averaging, so that the mean-square scattering amplitude is given by

$$
\overline{<b^{2}(\mathbf{k}, \hat{\mathbf{u}})>}=N^{-1} \sum_{i}<b_{i}^{2}\left(\mathbf{k}, \hat{\mathbf{u}}_{i}\right)>
$$

The length of the scattering vector $\mathbf{k}$ is given by

$$
k=\frac{4 \pi n}{\lambda_{0}} \sin (\theta / 2)
$$

with $n$ the refractive index of the dispersion, $\lambda_{0}$ the wavelength of the light in vacuo, and $\theta$ the scattering angle. The scattering amplitude $b(\mathbf{k}, \hat{\mathbf{u}})$ of a single 
particle with orientation $\hat{\mathbf{u}}$ is

$$
b(\mathbf{k}, \hat{\mathbf{u}})=\frac{2 \pi n(\partial n / \partial \varrho)_{\mathrm{T}}}{\lambda_{0}^{2} r} f(\mathbf{k}, \hat{\mathbf{u}}) \sqrt{I_{0}}
$$

where $r$ is the distance between the scattering volume and the detector, $Q$ is the number density of the particles, and $f(\mathbf{k}, \hat{\mathbf{u}})$ describes the interference effect of light scattered within single particles, which for cylinders takes the form

$$
f(\mathbf{k}, \hat{\mathbf{u}})=\frac{2 J_{1}\left(1 / 2 k D \sqrt{1-\frac{(\mathbf{k} \cdot \hat{\mathbf{u}})^{2}}{k^{2}}}\right)}{1 / 2 k D \sqrt{1-\frac{(\mathbf{k} \cdot \hat{\mathbf{u}})^{2}}{k^{2}}}} \times \frac{\sin (1 / 2 L \mathbf{k} \cdot \hat{\mathbf{u}})}{1 / 2 L \mathbf{k} \cdot \hat{\mathbf{u}}}
$$

with $L$ the length and $D$ the diameter of the cylinder, and $J_{1}$ the Bessel function of the first kind of order 1 . Note that $f^{2}(\mathbf{k}, \hat{\mathbf{u}})$ is equal to the form factor $P(\mathbf{k}, \hat{\mathbf{u}})$ of a cylinder. ${ }^{3}$ For infinitely thin rods, the first quotient on the right-hand side of eq 6 is 1 and $f(\mathbf{k}, \hat{\mathbf{u}})$ is given by the second quotient involving the sine function. The refractive index increment $(\partial n / \partial \varrho)_{\mathrm{T}}$ can be expressed in terms of the refractive indices of the particle $\left(n_{p}\right)$, which is assumed to be isotropic, and the solvent $\left(n_{\mathrm{m}}\right)$

$$
(\partial n / \partial \varrho)_{\mathrm{T}} \simeq V_{\mathrm{p}}\left(n_{\mathrm{p}}-n_{\mathrm{m}}\right)
$$

where $V_{\mathrm{p}}$ is the volume of the particle. ${ }^{5}$ Equation 7 is only accurate for $\left|n_{\mathrm{p}}-n_{\mathrm{m}}\right| \ll 1$, which, however, is also a requirement for the validity of the RGD theory itself. 3,15

The normalized autocorrelation function of the scattered electric field amplitude $E$ is

$$
g_{\mathrm{E}}(k, \tau)=\frac{<E(k, 0) E^{*}(k, \tau)>}{<|E(k, 0)|^{2}>}=\frac{F^{\mathrm{M}}(k, \tau)}{S^{\mathrm{M}}(\mathrm{k})}
$$

with $F^{\mathrm{M}}(k, \tau)$ the measured dynamic structure factor given by

$$
\begin{aligned}
& F^{\mathrm{M}}(k, \tau)=\left[N<b^{2}(\mathbf{k}, \hat{\mathbf{u}})>\right]^{-1} \times \\
& \sum_{i} \sum_{j}<b_{i}\left[\mathbf{k}, \hat{\mathbf{u}}_{i}(0)\right] b_{j}\left[\mathbf{k}, \hat{\mathbf{u}}_{j}(\tau)\right] \exp \left[i \mathbf{k}\left[\mathbf{r}_{i}(0)-\mathbf{r}_{j}(\tau)\right]\right]>
\end{aligned}
$$

Note that $F^{\mathrm{M}}(k, 0)=S^{\mathrm{M}_{(}}(k)$.

2.2. Static Light Scattering of an Assembly of Rods. Monodisperse Rods. It is not possible to separate the orientation dependent scattering amplitudes $b_{i}$ and $b_{j}$ from the position dependent exponential in the ensemble average in eq 2 , because the probability of finding particle $i$ at distance $\left|\mathbf{r}_{i}-\mathbf{r}_{j}\right|$ from particle $j$ depends strongly on the size and orientation of the particles. However, for identical particles with an isotropic refractive index, and in the limit of small scattering vectors $\mathbf{k}, b(\mathbf{k}, \hat{\mathbf{u}})$ is constant $(f(\mathbf{k}, \hat{\mathbf{u}})=1)$ and this separation can be made. For this special case, eq 2 reduces to

$$
S^{\mathrm{M}}(k=0)=S(k=0)=k_{\mathrm{B}} T\left(\frac{\partial \varrho}{\partial \Pi}\right)_{\mathrm{T}}
$$

where $k_{\mathrm{B}}$ is the Boltzmann constant, $T$ is the absolute temperature, and $\Pi$ is the osmotic pressure. Using eq 10 , the scattered intensity can be calculated from the osmotic compressibility, which can be obtained conveniently within the scaled particle theory for a system of hard spherocylinders (i.e., cylinders of length $L$ and diameter $D$, capped at both ends with a semisphere), giving:

$$
\begin{gathered}
k_{\mathrm{B}} T\left(\frac{\partial \varrho}{\partial \Pi}\right)_{\mathrm{T}}=\frac{(1-\varphi)^{4}}{\left(1+C_{1} \varphi\right)^{2}-C_{2} \varphi^{2}} \\
C_{1}=\frac{3 \gamma^{2}+1}{3 \gamma-1} \quad C_{2}=\frac{9 \gamma^{2}(\gamma-1)^{2}}{(3 \gamma-1)^{2}}
\end{gathered}
$$

where $\gamma=(L+D) / D$. It is known that the osmotic compressibility of a dispersion of hard repulsive spheres decreases so strongly with increasing concentration that the scattered intensity of a dispersion of repulsive spheres goes through a maximum as a function of the concentration. ${ }^{6}$ On the basis of eq 11 , we expect a similar behavior for the scattering of rodlike dispersions.

For larger scattering vectors, only second virial theories provide analytical expressions. ${ }^{16-20}$ These theories are considered to be rather good only for systems of very long and thin rods up to the concentration of the isotropic nematic phase separation.

Polydisperse Rods. The structure factor for rods polydisperse in length can also be calculated using a second virial approach. ${ }^{21}$ The restriction concerning the aspect ratio is similar as for the monodisperse case, and in the remaining part of this section, we will focus on scattering in the limit of small scattering vectors, where recently theoretical results for polydisperse rods were presented $^{14}$ (see section 2.4) which are valid up to high concentrations. These results were derived on the basis of the scaled particle theory and showed an intensity maximum for polydisperse rods as well.

For polydisperse particles, not only (collective) concentration fluctuations (or, more precise, fluctuations accompanied by variations in the osmotic pressure) give rise to refractive index variations but also exchange fluctuations from particles of different size and/or refractive index (without fluctuations in the osmotic pressure). Still, for polydisperse particles, the intensity can go through a maximum with increasing concentration, as was demonstrated for spheres already some time ago. ${ }^{22}$

2.3. Dynamic Light Scattering of a Dilute Assembly of Rods. We first consider a dilute isotropic dispersion of monodisperse rods, for which the dynamic light scattering is composed of a contribution due to translational and rotational diffusion. The translational diffusion is always observed, but whether rotational diffusion gives a contribution depends on the size and refractive index of the rods. The three mechanisms by which rotation can contribute to the DLS are outlined in the next paragraphs.

If $k L$ is large enough, the form factor will depend on the orientation, and rotation of the particle will give a variation in the scattered intensity. However, if we are interested in the scattered intensity in the limit of small scattering angles, the form factor equals one for all orientations, and this mechanism does not contribute to the DLS. ${ }^{23}$

The second mechanism also becomes important for large enough $k L$ but has a completely different origin. The basis of this mechanism is the coupling between rotational and translational diffusion, which is caused by the anisotropy of the translational diffusion coefficient. ${ }^{24}$

The last mechanism depends on the refractive index of the rods $\left(n_{\mathrm{p}}\right)$ relative to that of the solvent $\left(n_{\mathrm{m}}\right)$ which determines the importance of the intrinsic and form birefringence. ${ }^{25}$ The effect of the form birefringence is 
negligible if the difference between the (average) $n_{\mathrm{p}}$ and $n_{\mathrm{m}}$ is small, which is also a requirement for the accuracy of the RGD approximation. In fact, form birefringence is absent within the RGD approximation. ${ }^{15}$ The intrinsic birefringence,${ }^{24}$ however, makes the rotation important in DLS measurements with vertically polarized incident and scattered light, if the anisotropy of $n_{\mathrm{p}}$ is comparable to or larger than the difference between the average $n_{\mathrm{p}}$ and $n_{\mathrm{m}}$.

All three mechanisms (form birefringence excluded) are incorporated in the general theory of DLS from dilute systems of cylindrically symmetric particles by Aragón and Pecora. ${ }^{26}$ Their theory is exact within the RGD approximation.

If we rule out the contributions of rotation, the DLS of a dilute dispersion of rods is determined by the orientationally averaged translational diffusion. The measured normalized intensity autocorrelation function $g_{\mathrm{I}}(k, \tau)$ is then simply given by

$$
\begin{aligned}
g_{\mathrm{I}}(k, \tau)=\frac{\langle I(0) I(\tau)>}{<I>^{2}}=1+\left[g_{\mathrm{E}}(k, \tau)\right]^{2}=1+ \\
{\left[\mathrm{e}^{-k^{2} D_{0} \tau}\right]^{2} }
\end{aligned}
$$

where $\tau$ is the correlation delay time, $g_{\mathrm{E}}(k, \tau)$ is the normalized autocorrelation function of the scattered field amplitude, and $D_{0}$ is the orientationally averaged translational diffusion coefficient. Including polydispersity effects now yields

$$
g_{\mathbf{I}}(k, \tau)=1+\left[\sum_{j} \frac{<I_{j}>}{<I>} \mathrm{e}^{-k^{2} D_{j} \tau}\right]^{2}
$$

with $D_{j}$ the diffusion coefficient of component $j$ and $\left\langle I_{j}\right\rangle$ the average scattered intensity of component $j$. The orientationally averaged diffusion coefficient $D_{0}$ is given by Broersma ${ }^{27}$ as a function of the length $L$ and diameter $D$,

$$
D_{0}=\left(k_{\mathrm{B}} T / 3 \pi \eta_{0} L\right)(\ln (L / D)+v)
$$

with $\eta_{0}$ the viscosity of the solvent and $v$ a numerical correction for "end effects", for which we use the result obtained by Tirado and García de la Torre, ${ }^{28}$

$$
v=0.312+0.565(L / D)^{-1}-0.100(L / D)^{-2},(L / D \geq 2)
$$

2.4. Dynamic Light Scattering of a Concentrated Isotropic Assembly of Rods. For higher concentrations, no rigorous theory is known, and the existing approximate theories are mainly derived for stiff polymers, i.e., rods with a large length over width ratio. $7,9,16-19,24,29-32$ These theories are not complete, as they often focus on a specific effect in a certain concentration range. However, certain aspects of polydispersity, which are important in the present study, are not discussed in these papers. These specific aspects are discussed in what follows.

First, we will consider the simple case of rods with only optical polydispersity, but monodisperse in size, with an isotropic refractive index and in the limit of small scattering vectors $\mathbf{k}$. For an assembly of these rods, there is no correlation between the scattering amplitudes $b_{i}$ and $b_{j}$ and the position vectors $\mathbf{r}_{i}$ and $\mathbf{r}_{j}$. Therefore, eq 9 can be separated into two modes (describing the collective and self-diffusion), in a similar way as for spheres, ${ }^{2,12}$ leading to

$$
F^{\mathrm{M}}(k, \tau)=\frac{\bar{b}^{2}}{\overline{b^{2}}} F(k, \tau)+\left[1-\frac{\bar{b}^{2}}{\overline{b^{2}}}\right] F_{\mathrm{S}}(k, \tau)
$$

with

$$
\begin{array}{r}
F(k, \tau)=N^{-1} \sum_{i} \sum_{j}<\exp \left[i \mathbf{k} \cdot\left[\mathbf{r}_{i}(0)-\mathbf{r}_{j}(\tau)\right]\right]>= \\
S(k=0) \mathrm{e}^{-k^{2} D_{\mathrm{c}} \tau}
\end{array}
$$

the full dynamic structure factor and

$$
F_{\mathrm{S}}(k, \tau)=<\exp [i \mathbf{k} \cdot(\mathbf{r}(0)-\mathbf{r}(\tau)]]>=\mathrm{e}^{-k^{2} D_{\mathrm{s}} \tau}
$$

the self-dynamic structure factor. Here $D_{\mathrm{c}}$ is the collective diffusion coefficient, $D_{\mathrm{s}}$ is the long-time selfdiffusion coefficient, and $S(k=0)$ is the monodisperse structure factor as defined in eq 10 . Note that (for small scattering vector) $b$ no longer depends on the orientation vector $\hat{\mathbf{u}}$ nor the scattering vector $\mathbf{k}$

For rods polydisperse in size, the separation given by eq 15 can not be made rigorously anymore. However, the same arguments as used for dispersions of spherical particles ${ }^{12}$ can be applied to the system of rods in the limit of small scattering vectors, leading to a measured autocorrelation function that, to a good approximation, can be separated in two similar modes,

$$
F^{\mathrm{M}}(k, \tau) \simeq \frac{\bar{b}^{2}}{\overline{b^{2}}} S(k=0) \mathrm{e}^{-k^{2} D_{-\tau} \tau}+A_{-} \mathrm{e}^{-k^{2} D_{-} \tau}
$$

where $D_{+}$is associated with the "average" collective diffusion coefficient and $D_{-}$is associated with the "average" self- (or exchange) diffusion coefficient. Note that the two modes are not strictly single exponential, but some average over a polydisperse distribution of exponents. The mode amplitude obtained for the selfdiffusion mode of the optically polydisperse system in eq 15 , however, is not a good approximation for the

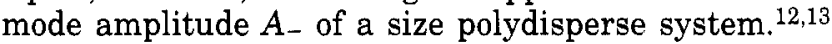
This is due to the fact that the scattering amplitude for particles with a different size is proportional to the volume of the particle, whereas the scattering amplitudes for optical polydisperse particles is independent of the particle size. Therefore, in the size polydisperse system, the interparticle distance and the scattering amplitudes are correlated, leading to a different mode amplitude. Combining now eqs 1 and 18 for $\tau=0$ leads to

$$
\langle I\rangle \simeq\left\langle I^{+}\right\rangle+\left\langle I^{-}\right\rangle
$$

where

$$
<I^{+}>=N \bar{b}^{2} S(k=0),<I^{-}>=N \overline{b^{2}} A_{-}
$$

and with eq 8 one finds,

$$
g_{\mathrm{E}} \simeq \frac{\left\langle\Gamma^{+}>\right.}{<I>} \mathrm{e}^{-k^{2} D_{+} \tau}+\frac{<I^{-}>}{<I>} \mathrm{e}^{-k^{2} D_{-} \tau}
$$

The two dynamic modes correspond to the collective and exchange fluctuations, as discussed in the static lightscattering part. The self-diffusion coefficient is expected to decrease strongly with increasing concentration, 7,33 whereas for the collective diffusion coefficient indications have been found for a moderate increase of $D_{c}$ with concentration, as well as a small decrease of $D_{\mathrm{c}}$ with the concentration. ${ }^{7,9}$ Independent of the precise concentration dependence, however, it is expected that for higher concentrations, the diffusion coefficients will 
differ sufficiently to distinguish the two modes in the measured autocorrelation function.

It is noted that at high concentrations there may still be additional modes due to, for instance, rotation translation coupling of individual rods ${ }^{24,29}$ and/or pretransitional fluctuations on approaching the isotropic nematic phase transition. ${ }^{18}$ In our experiments, however, we do not observe these modes.

The intensities $\langle I\rangle$ and $\left\langle I^{+}\right\rangle$for a polydisperse distribution of hard spherocylinders (cylinders of length $L$ and diameter $D$, capped at both ends with hemispheres) are calculated from a recently extended version of the scaled particle theory. ${ }^{14}$ The expressions obtained in the SPT for arbitrary hard convex particles can be described in terms of four "fundamental measures", ${ }^{34}$ which for hard spherocylinders are defined by

$$
\begin{gathered}
R_{k}^{(0)}=1, R_{k}^{(1)}=1 /{ }_{2} D_{k}+1 /{ }_{4} L_{k} \\
R_{k}^{(2)}=\pi D_{k}^{2}+\pi D_{k} L_{k}, R_{k}^{(3)}=1 /{ }_{6} \pi D_{k}^{3}+1 /{ }_{4} \pi D_{k}^{2} L_{k}
\end{gathered}
$$

where $k$ denotes the labeling number of the particle. These fundamental quantities have a simple meaning: $R_{k}^{(1)}$ is the radius of curvature integrated over the surface, $R_{k}^{(2)}$ is the surface area, and $R_{k}^{(3)}$ is the volume of the particle. These single particle quantities are used to define the averaged quantities,

$$
\xi^{(\prime)}=\sum_{k=1}^{N} \varrho_{k} R_{k}^{(v)} \text { and } \xi^{(1, k)}=\sum_{k=1}^{N} \varrho_{k} R_{k}^{(j)} R_{k}^{(\mu)}
$$

in terms of which the scattered intensity at zero scattering angle is given as

$$
\begin{aligned}
& \langle I\rangle_{\forall=0}=\left(I_{0} V_{s} / r^{2}\right) \frac{4 \pi^{2} n^{2} A^{2}}{\lambda_{0}^{4}}(1-\varphi)^{2}\left[\xi^{(3,3)}+\right. \\
& \left.\frac{\xi^{(2.2)}\left(\xi^{(1,3)}\right)^{2}-2\left(1-\varphi+\xi^{(1.2)}\right) \xi^{\left(1.3 \xi^{(2,3)}\right.}+\left(\xi^{(1.1)}-\xi^{(2)} / 4 \pi\right)\left(\xi^{(2.3)}\right)^{2}}{\left(1-4+\xi^{(1.2)}\right)^{2}-\left(\xi^{(1,1)}-\xi^{(2)} / 4 \pi\right) \xi^{(2.2)}}\right]
\end{aligned}
$$

Here $V_{\mathrm{s}}$ is the scattering volume, $A=\left(\mathrm{d} n / \mathrm{d} \underline{g}_{i}\right) / R_{i}^{(3)} \simeq n_{\mathrm{p}}$ $-n_{\mathrm{m}}$, and $\varphi$ is the volume fraction. As discussed in the previous paragraph, the scattered intensity is composed of a part due to overall concentration fluctuations (accompanied by osmotic pressure fluctuations) and a part due to exchange (or polydispersity) fluctuations. The former part can be calculated separately from the osmotic compressibility, leading to

$$
\begin{aligned}
& <I^{+}>= \\
& \left(I_{0} V_{s} / r^{2}\right) \frac{4 \pi^{2} n^{2} \lambda_{0}^{-4} A^{2} \varphi^{2}(1-\varphi)^{4}}{\xi^{(0)}(1-\varphi)^{2}+2 \xi^{(1)} \xi^{(2)}(1-\varphi)+\left(\xi^{(2)}\right)^{3} / 4 \pi}(24)
\end{aligned}
$$

We note here the square above the $\varphi$ in the numerator, which is missprinted in the original paper. ${ }^{14}$ The description of the size distribution of the particles is given in the Appendix.

\section{Experimental Section}

Particle Synthesis. Colloidal boehmite rods were synthesized according to Buining ${ }^{10}$ by adding $91.5 \mathrm{~g}$ of aluminum trisec-butoxide to $2 \mathrm{~L}$ of $0.037 \mathrm{M} \mathrm{HCl}$ while stirring. After 3 days of continuous stirring, the turbid liquid was treated hydrothermally at $150{ }^{\circ} \mathrm{C}$ for $20 \mathrm{~h}$. This hydrothermal treatment was performed without the usual rotation of the autoclaves, and the autoclaves were left at rest for about $90 \mathrm{~min}$ (this probably allows some sedimentation) before putting them in the oven. This seemingly minor change with respect to
Buinings prescription ${ }^{10}$ turned out to give an important reduction of the degree of clustering. The reduced clustering was demonstrated by comparing the electron microscopy (EM) and SLS results, as outlined in the next section. After the hydrothermal treatment, the dispersion was dialyzed against flowing demiwater for 13 days.

The grafting of the bare boehmite particles was performed according to Buining, ${ }^{11}$ with the following concentrations. The boehmite concentration during the distillation step in which the particles are transferred to propanol was kept below 1.6 $\mathrm{g} / \mathrm{L}$. Care was taken that the level of the liquid stayed above the heated part of the distillation vessel, to prevent rapid drying of liquid films. To $3.5 \mathrm{~L}$ of propanol sol was added 2.8 $\mathrm{L}$ of a solution of $10 \mathrm{~g}$ of modified poly(isobutene) in $1 \mathrm{~L}$ of tetrahydrofurane. Stepwise addition of toluene and distillation yielded a dispersion in toluene, which was then concentrated to about $10 \mathrm{~g} / \mathrm{L}$. After cooling, the dispersion was exposed to ultrasonication for $15 \mathrm{~min}$, resulting in a reduction of the turbidity of the dispersion. After 2 days, the excess modified poly(isobutene) was removed by two steps consisting of ultracentrifugation at $10000 \mathrm{rpm}$ followed by redispersion in cyclohexane. Accumulated dust was removed by $2 \mathrm{~h}$ of centrifugation at $2000 \mathrm{rpm}$, yielding a stable, dark red (by absorbance) stock dispersion of $9.39 \mathrm{w} / \mathrm{w} \%$. The last concentration step and the change of solvent to o-dichlorobenzene are described in section 4 .

Electron Microscopy and Density Determination. Electron microscopy photographs were made with a Philips CM10 transmission microscope. The samples were prepared by spraying a very dilute dispersion on 300 mesh copper grids coated with a thin carbon film. The thin carbon film was not stabilized by a polymer film to reduce the background and thereby increase the contrast in the electron microscope. In this way, enough contrast was obtained without having to work out of focus.

The particle density was determined by weighing $10 \mathrm{~mL}$ of a $9.39 \mathrm{w} / \mathrm{w} \%$ dispersion in cyclohexane and $10 \mathrm{~mL}$ of cyclohexane in the same measuring flask, after which the density of the particles can simply be calculated. The weight/ weight concentration is determined by weighing a small amount of dispersion and then drying to constant weight under an IR lamp.

Static and Dynamic Light Scattering. The static and dynamic light-scattering measurements were performed on a laboratory-built laser light-scattering setup at $647 \mathrm{~nm}$ using vertically polarized incident and scattered light. All measurements were performed at $25^{\circ} \mathrm{C}$. Prior to each measurement, samples were centrifuged at $2000 \mathrm{rpm}$ from $5 \mathrm{~min}$, for the most dilute sample, to $20 \mathrm{~min}$, for the most concentrated sample, in order to remove dust. Under these conditions, no significant particle sedimentation occurred.

Although the SLS measurement was somewhat less accurate than on a dedicated SLS setup, because of the small scattering volume that was necessary for the DLS measurement, this did not seriously disturb our results. To optimize the SLS measurement, the cuvette was rotated during the measurement, so that irregularities on the cuvette and slow dynamical fluctuations of the scattered light at high concentrations were averaged out. Furthermore, a pinhole in front of the detector was optimized for maximum intensity when necessary, to prevent a possible error in the alignment of the laser and the detector on changing the scattering angle. All SLS measurements were corrected for solvent scattering, and a simple transmission correction was applied by division of the measured intensity by the transmission of the dispersion (it is noted that the particles also absorb light). The intensities were calibrated using a reference sample. Autocorrelation functions were obtained with a 128 channel, 8 bit hardware correlator (Malvern) in combination with a software correlator for the slowly decaying functions.

\section{Characterization and Dispersion Properties}

Dispersion Properties. The stock dispersion in cyclohexane at a concentration of $9.39 \mathrm{w} / \mathrm{w} \%$ was concentrated by evaporation at room temperature, until 


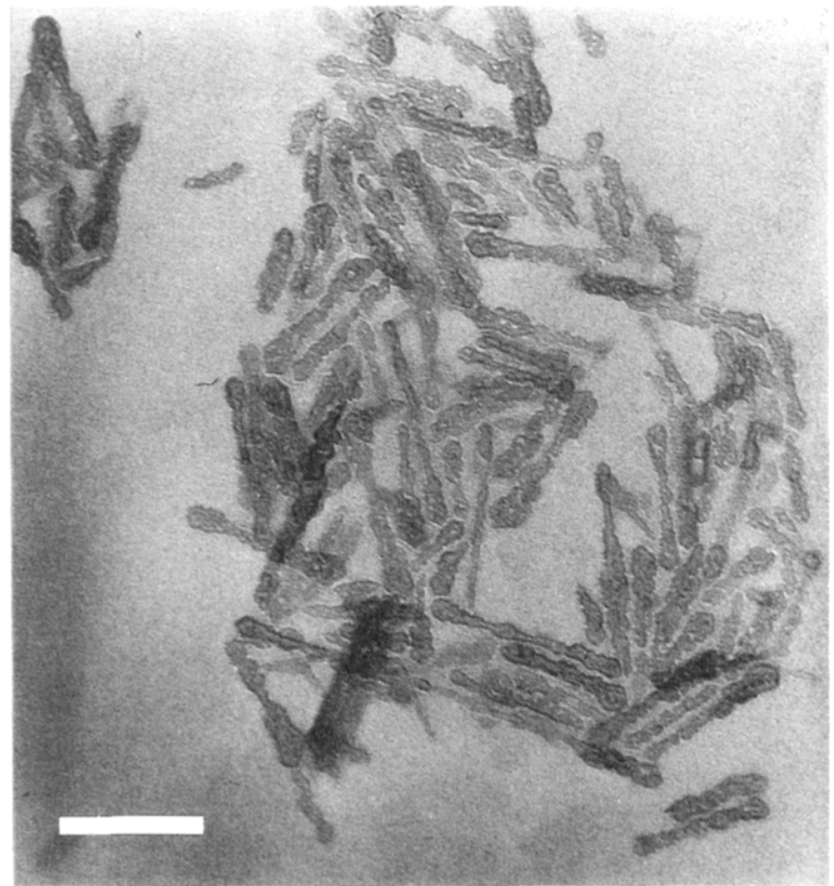

Figure 1. Electron microscopy photograph of the grafted boehmite rods. The bar denotes $100 \mathrm{~nm}$.

a very viscous dispersion was obtained. The concentrated sample was stirred for at least 2 weeks, after which $o$-dichlorobenzene (DCB) was added. Evaporation of the cyclohexane yields a dispersion in DCB.

The above described treatment was first performed on a small test sample (ASB33g-1), after which a larger amount of dispersion was used (ASB33g-2). Three months later, a third (and the last) amount of sample was concentrated and transferred to DCB (ASB33g-3). Although the three batches were treated in as much the same way as possible, a large difference in dispersion behavior was observed. The first two dispersion batches, ASB33g-1 and -2, start to form gel-like clots at a volume fraction of about 18\%, whereas ASB33g-3 remained homogeneous up to a volume fraction of about $28.5 \%$, after which an isotropic nematic phase separation occurs. The origin of this completely different behavior is still unclear. Further experiments focus on ASB33g3 .

At a concentration of $29.2 \mathrm{v} / \mathrm{v} \%$, a phase separation was observed in an isotropic phase of $28.5 \pm 0.3 \mathrm{v} / \mathrm{v} \%$ and a nematic phase of $31 \pm 1 \mathrm{v} / \mathrm{v} \%$. The phase separation is completed in about 2 days. It was striking that after stirring the quiescent dispersion exhibits an almost steady birefringence pattern for several hours, as was observed between crossed polars by eye, before macroscopic phase separation occurs. The nematic phase is very viscous; it took about $10 \mathrm{~min}$ to flow completely after the vessel was tilted, whereas the upper isotropic phase flowed immediately.

Electron Microscopy and Particle Density. Electron microscopy photographs (see example in Figure 1) were taken to determine the size and shape distribution of the particles. Figure 1 shows that the particles have an irregular structure. The length and diameter of the particles are determined with an interactive image analysis system (IBAS). For particles with an irregular structure, the diameter was estimated. Figure 2 shows a scatter plot of the measured lengths and diameters. The average dimensions with their standard deviations and the correlation coefficient are given in Table 1. There is a weak but significant correlation between

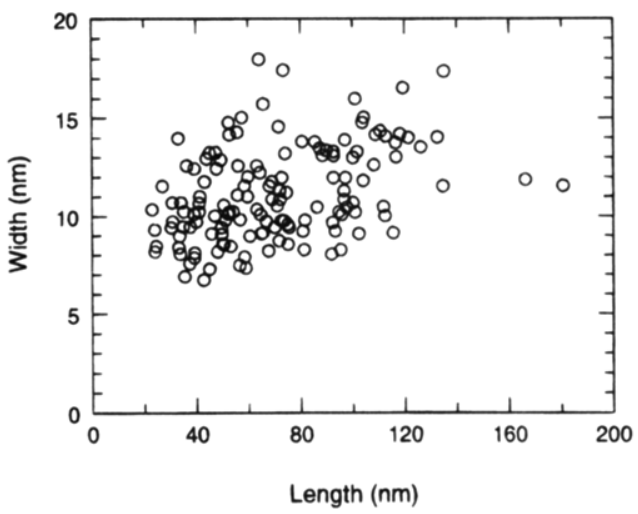

Figure 2. Scatter plot showing the length and width of the particles measured from electron microscopy photographs.

Table 1. Average Particle Dimensions as Determined from Electron Microscopy for 142 Particles

$\begin{array}{llll}\bar{L} & 71 \mathrm{~nm} & \sigma_{\mathrm{D}} & 2.4 \mathrm{~nm} \\ \sigma_{\mathrm{L}} & 31 \mathrm{~nm} & r(L, D) & 0.40 \\ \bar{D} & 11.1 \mathrm{~nm} & & \end{array}$

length and width, which is also seen in Figure 2. Note that the actual diameter in the dispersion may deviate somewhat from the EM diameter because of swelling of the steric stabilization layer.

The average density of the particles is $1.97 \pm 0.07 \mathrm{~g}$ $\mathrm{mL}^{-1}$. This density is used to calculate the volume fractions from the measured weight/weight concentrations.

\section{Static Light Scattering and Particle Clustering}

Experiments on previous systems already showed the existence of clusters in poly(isobutene)-grafted boehmite dispersions. ${ }^{35}$ In these experiments, the clusters were analyzed by a combination of EM, SLS on dilute samples, and series filtrations using filters with different pore sizes. An important conclusion was that the degree of clustering can be evaluated semiquantitatively from EM and SLS. More precisely, the degree of clustering was shown to increase with an increasing difference between the measured SLS curve and a calculated curve based on the EM dimensions. Unfortunately, the filtrations could only be used to remove small amounts of clusters because the filters stop up after a short filtration time.

In the new synthesis of ASB33, the clustering has been minimized by evaluating the degree of clustering at several points in the synthesis and then selecting the best result. There are two instances at which the clustering could be reduced. The first cluster reduction has already been described in the synthesis part of the Experimental Section. The second reduction of the degree of clustering could be achieved in the step in which the grafted dispersion is concentrated by evaporation of the solvent. Taking a series of samples during the evaporation process revealed that vigorous stirring of a concentrated viscous dispersion gives a reduction of the clustering. Stirring at intermediate concentrations did not make any difference. The separate effect of evaporation is not clear, as this is always combined with stirring. Figure 3 shows the remaining difference between the measured SLS curve and the calculated curve based on the EM dimensions from Table 1, clearly indicating some clustering (see section 6.1 for details on the calculated curve). Unfortunately, we were not able to reduce the clustering to a negligible level. 


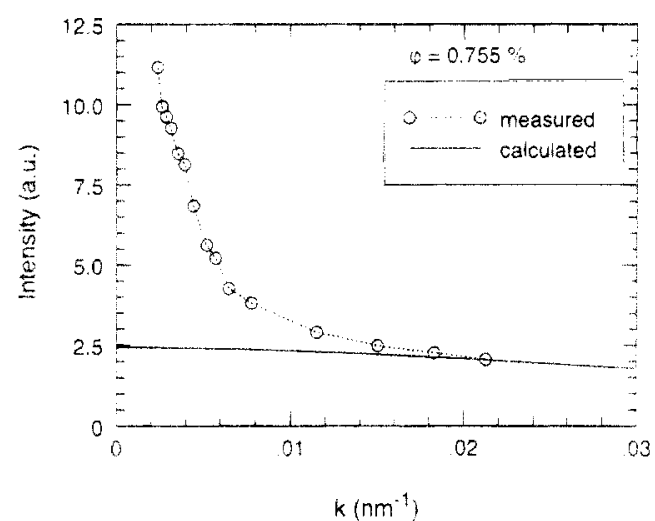

Figure 3. Total scattered intensity of the $0.755 \mathrm{v} / \mathrm{v} \%$ dispersion compared to a calculated scattering curve based on the EM dimensions. The intensity of the calculated curve is scaled to go through the last measured point.

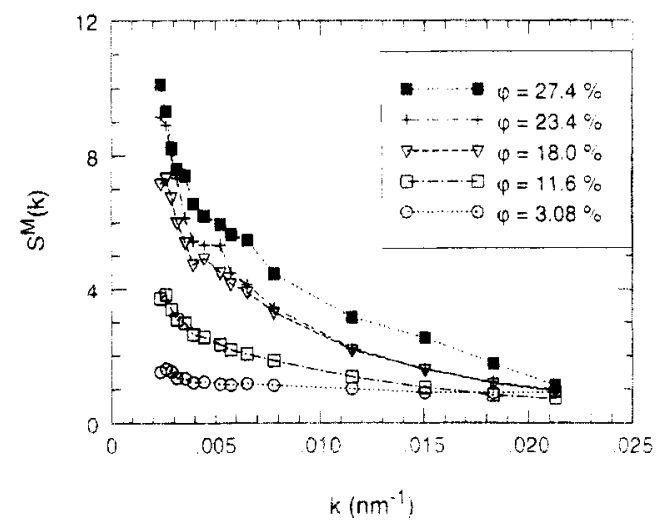

Figure 4. Measured structure factor for the total scattering intensity (rods + clusters) as a function of $k$ for five concentrations. The symbols denote the measured points. The 0.755 $\mathrm{v} / \mathrm{v} \%$ sample is used as the dilute (form factor) sample in the calculation of $S^{\mathrm{M}}$

The structure factor $S^{\mathrm{M}}(k)$ as obtained from the ratio:

$$
S^{\mathrm{M}}(k)=\frac{<I(k)>/ \varphi}{<I(k)>/ \varphi_{1}}
$$

with $\varphi_{1}$ a small volume fraction at which interactions between the rods are neglectable (we used $\varphi_{1}=0.755$ $\mathrm{v} / \mathrm{v} \%)$ and $\langle I(k)\rangle_{1}$ the corresponding intensity, is shown in Figure 4. $S^{\mathrm{M}_{(}}(k)$ shows an upswing at small scattering angles, which increases with the concentration, reaching values much higher than one. This strong increase of $S^{\mathrm{M}}(k)$ is reversible, i.e., if a concentrated dispersion is diluted, the scattering curve corresponding to the lower concentration is recovered. Upon further dilution of the $0.755 \mathrm{v} / \mathrm{v} \%$ sample, however, down to concentrations of $0.069 \mathrm{v} / \mathrm{v} \%$, a constant $S^{\mathrm{M}}(k)$ remained.

In general, the described behavior of $S^{\mathrm{M}}(k)$ indicates an attractive interaction in the dispersion. However, the dynamic light-scattering analysis to be described in section 6 shows that the increasing $S^{M_{(}}(k)$ is completely due to additional clustering. Therefore, the strong increase of $S^{\mathrm{M}}$ at small scattering vectors is interpreted as an increased cluster size, and possibly also partly by an increased cluster concentration. It is noted that a significant rod-rod aggregation is not probable, as the results to be described in section 6 are consistent with a repulsive rod-rod interaction.

It is not possible to estimate the concentration of the clusters directly from the scattering data. The scattering of a cluster depends (apart from its size) also on its shape, refractive index, and whether or not the cluster has a more or less compact structure. For compact, thick, rodlike or spherical clusters, only a relatively low volume fraction of clusters will be needed to explain the cluster scattering. However, the clusters may be less compact, and we can only say that the results are consistent with a moderate cluster concentration.

However, it is possible to make a rough estimate of the cluster size as well as its increase with the concentration by combining the SLS curves given in Figures 3 and 4 with the dynamic mode amplitudes of the rods and clusters which will be discussed in section 6 . This procedure allows the determination of the scattered intensity of the clusters by subtracting the intensity due to the rods. These cluster scattering curves can be used to determine the Guinier radius ${ }^{3,4}\left(R_{\mathrm{g}}\right)$ from a plot of $\ln \left(\langle I(k)>)\right.$ against $k^{2}$, where the initial slope gives $R_{g}^{2} / 3$. This results in Guinier radii of 350 and $450 \mathrm{~nm}$ for the 0.755 and $3.06 \mathrm{v} / \mathrm{v} \%$ sample, respectively. For larger volume fractions, it was not possible to extract the Guinier radius from the experimental data. However, an estimate of the relative increase in cluster size can be obtained from the absolute scattered intensity of the clusters extrapolated to $k=0$. To do this, the cluster volume fraction and the density and shape of a cluster are assumed to be constant, so that the estimated intensity at $k=0$ is directly proportional to $R_{g}^{3}$ of the cluster. This results in an increase in cluster size which is in good agreement with the results from the Guinier radius for the two volume fractions of 0.755 and $3.06 \mathrm{v} / \mathrm{v} \%$. For the larger volume fractions, a cluster size up to 2.8 times the size of that in the $0.755 \mathrm{v} / \mathrm{v} \%$ sample is obtained, resulting in a maximum $R_{\mathrm{g}}$ of 980 $\mathrm{nm}$.

The next section (6.1) deals with the separation of the scattered light of a low-concentration sample in a part due to the clusters and in a part due to the rods.

\section{Combined Static and Dynamic Light Scattering}

6.1. Scattering from a Dilute Dispersion. The lowest concentration for which both SLS and DLS measurements were performed is $0.755 \mathrm{v} / \mathrm{v} \%$, corresponding to a number concentration of $0.351 / \mathrm{L}^{3}$, which is close to the limit of noninteracting rods. For smaller concentrations, the scattered intensity is too low for an accurate DLS measurement.

The possible mechanisms by which rotation can affect the dynamic light scattering were already outlined in the theoretical section. As the maximum scattering vector in our experiments is $0.0213 \mathrm{~nm}^{-1}$, and given the dimensions of the rods in Table 1, it can be concluded that the first two mechanisms mentioned in the theoretical section are not important ${ }^{23,24}$ (contributions are less than $1 \%$ ). Only the mechanism related to the intrinsic birefringence can not be excluded rigorously because we only have crude estimates of the relevant experimental parameters. A rough estimate of the average refractive index difference, $n_{\mathrm{p}}-n_{\mathrm{m}}$ (based on the absolute scattered intensity), yields a value of 0.015 , which is of the same order of magnitude as the refractive index anisotropy of a pure boehmite crystal..$^{36}$ From ref 24 , however, it can be shown that rotation has an appreciable effect only if the anisotropy in the refractive index is larger than the difference $n_{\mathrm{p}}-n_{\mathrm{m}}$ for our maximum $k L$. This indicates that rotation due to the last mechanism described in the theoretical section may also be neglected.

The measured field autocorrelation function $\left(g_{\mathrm{E}}\right)$ was found to consist of two exponentials, as is illustrated 


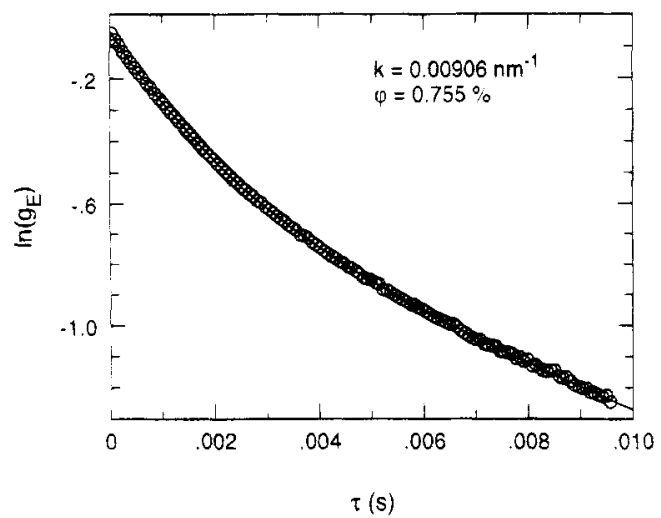

Figure 5. Logarithmic plot of the measured field autocorrelation function for the $0.755 \mathrm{v} / \mathrm{v} \%$ sample at $k=0.00906 \mathrm{~nm}^{-1}$ (O) compared to a two-exponential fit (drawn line).

Table 2. Diffusion Coefficients and Relative Mode Amplitudes Resulting from a Double-Exponential Fit of $g_{E}$ for the $0.755 \mathrm{v} / \mathrm{v} \%$ Sample

\begin{tabular}{cccc}
\hline $\begin{array}{c}k \\
\left(\mathrm{~nm}^{-1}\right)\end{array}$ & $\left\langle I_{\text {rod }}>/<I>\right.$ & $\begin{array}{c}D_{\text {rod }} \\
\left(10^{6} \mathrm{~nm}^{2} \mathrm{~s}^{-1}\right)\end{array}$ & $\begin{array}{c}D_{\text {cluster }} \\
\left(10^{6} \mathrm{~nm}^{2} \mathrm{~s}^{-1}\right)\end{array}$ \\
\hline 0.00575 & 0.214 & 6.5 & 0.92 \\
0.00677 & 0.315 & 6.0 & 0.82 \\
0.00906 & 0.426 & 5.9 & 0.83 \\
0.01152 & 0.495 & 5.4 & 0.55 \\
0.01506 & 0.582 & 5.5 & 0.67 \\
0.02129 & 0.541 & 7.0 & 1.8
\end{tabular}

for the measurement at $k=0.00906 \mathrm{~nm}^{-1}$ in Figure 5 . Clearly, the double exponential gives a very good description of the measured $g_{\mathrm{E}}$. It is noted that the actual nonlinear least-square curve fits have been made on $g_{1}$, using eq 13 with two exponents. Results of the fits at six different scattering angles are shown in Table 2. All six fits are of similar quality. The slow decay of the correlation functions is attributed to large (permanent) clusters and the fast decay to single rods. The basis for this interpretation is given in the next two paragraphs.

The theoretical value of the orientationally averaged translational diffusion coefficient for the single rods is calculated on the basis of the EM dimensions in Table 1. This is done by calculation of $g_{1}$ from eq 13 , using the numerical integration over the distribution function from eq A1, and using the scattering amplitude from eqs 5 and 6 substituted in eq 1 to calculate the mode amplitudes $\left\langle I_{j}>\right|<I>$. This calculation is "exact" within the RGD approximation apart from the $L / D$ factor in eq 14 , which was set to its average value for efficiency reasons. Of course, these calculated autocorrelation functions deviate somewhat from single-exponential behavior, and the determination of an average diffusion coefficient from a single-exponential fit of $g_{I}$ is just an estimate that may be compared to the values listed in Table 2. Six average diffusion coefficients at six scattering vectors are calculated in this way, giving an overall average diffusion coefficient of $7.1 \times 10^{6} \mathrm{~nm}^{2} \mathrm{~s}^{-1}$, compared to a value of $10.5 \times 10^{6} \mathrm{~nm}^{2} \mathrm{~s}^{-1}$ for the monodisperse calculation obtained by simply substituting the average dimensions in eq 14. Comparing the average diffusion coefficient to the measured values of the fast mode in Table 2 (average $D$ is $6.1 \times 10^{6} \mathrm{~nm}^{2}$ $\mathrm{s}^{-1}$ ) shows a rather good agreement between the measured average diffusion coefficient and the calculated ones on the basis of the EM dimensions.

If the fast mode is due to single-rod scattering, then the scattered intensity of the rods can be obtained from the total scattered intensity multiplied by the relative

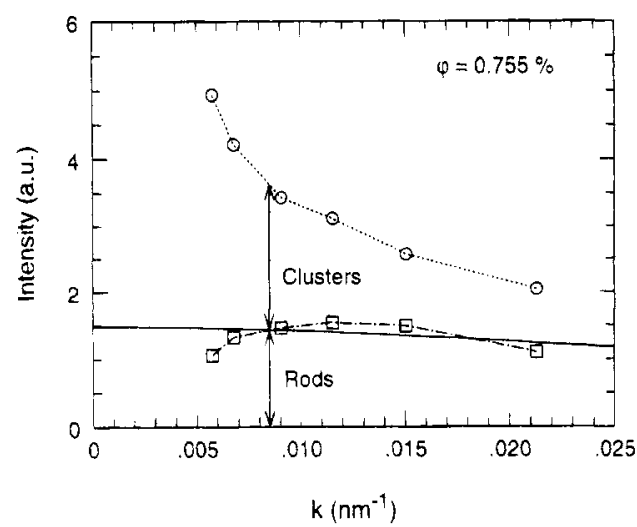

Figure 6. Scattered intensity as a function of $k$ for the 0.755 $\mathrm{v} / \mathrm{v} \%$ sample. The upper curve (O) gives the total intensity, and the lower dashed curve ( $\square$ ) shows the rods intensity calculated from the DLS mode amplitudes and the total intensity. The symbols denote the measured points; the lines are drawn to guide the eye. The drawn curve to $k=0$ is the scattering curve calculated on the basis of the EM dimensions. Its overall intensity is scaled by hand to fit the measurement.

mode amplitudes as given in Table 2 . Splitting up the scattered intensities in this way further confirms this interpretation, as is shown in Figure 6, where the scattered intensity of the rods is compared to a calculated light-scattering curve using the EM dimensions from Table 1 with the size distribution from eq $A 1$, in combination with eqs 1 and 3-6. Although the scattering curve of the rods is curved, the agreement is quite reasonable. The reason for the systematic curvature is not clear. It may be due to the limited accuracy of the amplitudes obtained from the DLS measurements. Another possible explanation could be found in the close refractive index matching of the particles.

It is striking that the clusters yield only one dynamical mode, which is fairly close to single exponential. The interpretation that the slowly decaying mode is indeed a cluster mode is supported by the agreement of the two resulting modes with theoretical SLS as well as DLS calculations. This separation is applied in the next section to light-scattering data of more concentrated samples.

6.2. Light Scattering as a Function of the Concentration. Observed Dynamical Modes. The measurements discussed in this section were performed at a relatively large wave vector $\left(k=0.0213 \mathrm{~nm}^{-1}\right)$ in order to minimize the cluster contribution to the scattering. Even at this high scattering vector the clusters make a substantial contribution. A representative example of the measured field autocorrelation functions at $k=$ $0.0213 \mathrm{~nm}^{-1}$ is shown in Figure 7 for the $23.4 \mathrm{v} / \mathrm{v} \%$ sample. The function is composed of three exponentials, of which the cluster component has by far the largest mode amplitude. Indeed, it is the cluster mode which dominates the complete plot of $g_{E}$ in Figure 7a. The enlarged first part of $g_{\mathrm{E}}$ in Figure $7 \mathrm{~b}$ shows the two faster modes due to the rods. Although, for this short time range the final slope is already dominated by the clusters (mode 3 ), the two modes corresponding to the rods can be distinguished clearly.

Table 3 contains a summary of the DLS results at $k$ $=0.0213 \mathrm{~nm}^{-1}$, as obtained from two or three exponential fits. At higher concentrations, the scattering of the rods separates into a fast and slow mode (mode 1 and 2 in Table 3), which are interpreted as a collective diffusion mode and an exchange diffusion mode, respectively, as given in eq 20. The third mode describes the cluster scattering. Note, that now $\langle I\rangle=\left\langle I^{+}\right\rangle+\left\langle I^{-}\right\rangle$ 

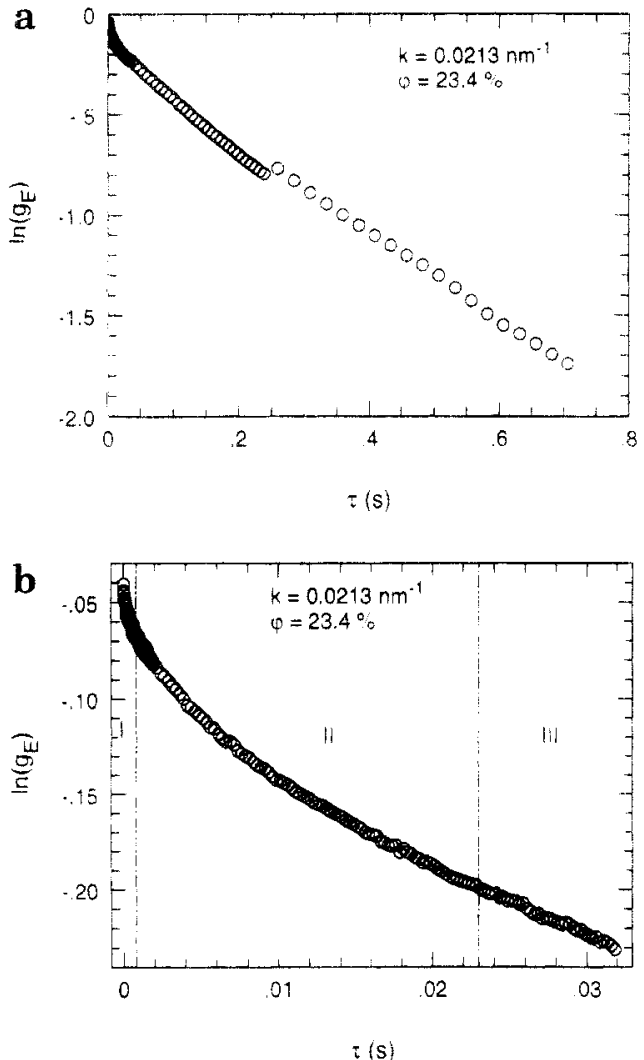

Figure 7. Logarithmic plot of the measured field autocorrelation function of the $23.4 \mathrm{v} / \mathrm{v} \%$ sample at $k=0.0213 \mathrm{~nm}^{-1}$. Panel a shows the complete measurement, which is dominated by cluster scattering, and panel $\mathrm{b}$ gives the enlarged beginning, together with a rough indication of the three modes.

Table 3. Mode Amplitudes and Diffusion Coefficients for the DLS Measurements at $k=0.0213 \mathrm{~nm}^{-1}$ as a Function of the Concentration

\begin{tabular}{ccccccc}
\hline $\begin{array}{c}Q \\
\mathrm{v} / \mathrm{v} \%)\end{array}$ & $\begin{array}{c}\left\langle I_{1}>/\right. \\
\langle I\rangle\end{array}$ & $\begin{array}{c}\left\langle I_{2}>/\right. \\
\langle I\rangle\end{array}$ & $\begin{array}{c}\left\langle I_{3}>\right. \\
\langle I\rangle\end{array}$ & $\begin{array}{c}D_{1}\left(10^{6}\right. \\
\left.\mathrm{nm}^{2} \mathrm{~s}^{-1}\right)\end{array}$ & $\begin{array}{c}D_{2}\left(10^{6}\right. \\
\left.\mathrm{nm}^{2} \mathrm{~s}^{-1}\right)\end{array}$ & $\begin{array}{c}D_{3}\left(10^{6}\right. \\
\left.\mathrm{nm}^{2} \mathrm{~s}^{-1}\right)\end{array}$ \\
\hline 0.755 & 0.54 & & 0.46 & \multicolumn{2}{c}{7.0} & 1.8 \\
3.08 & 0.52 & & 0.48 & \multicolumn{2}{c}{6.4} & 0.84 \\
11.6 & 0.13 & 0.24 & 0.63 & 8.5 & 1.44 & 0.092 \\
18 & 0.048 & 0.107 & 0.845 & 7.0 & 0.47 & 0.030 \\
23.4 & 0.0175 & 0.076 & 0.907 & 7.5 & 0.26 & 0.0062 \\
27.4 & 0.0072 & 0.041 & 0.952 & 5.5 & 0.156 & 0.0023
\end{tabular}

$+\left\langle I_{\text {cluster }}>\right.$ in eq 20 , with $\left\langle I_{\text {cluster }}>\right.$ the scattered intensity due to the additional cluster mode. The quality of the multiple exponential fits was checked by hand, by comparing the fit and the measured data directly and/or by residual plots, and a fit with an additional exponent was found to give no significant improvement of the fits.

The scattering vector $k=0.0213 \mathrm{~nm}^{-1}$ corresponds to $k \bar{L}=1.5$, with $\bar{L}$ the average length. On the basis of the second virial approximation for the static structure factor, ${ }^{16,20}$ we think that this value is sufficiently small to interpret experimental data in terms of zero wave vector theory.

Diffusion Coefficients. Within the uncertainty of its experimental determination of about $25 \%$, the diffusion coefficient of the fast collective mode shows hardly any concentration dependence, while the slow exchange diffusion mode decreases strongly with concentration, as is shown in Figure 8 . The trend of this behavior is similar to the concentration dependence that is found for spheres. $2,13,37-39$

Although hardly significant in our measurements, the small decrease in $D_{0}$ at the highest volume fraction is

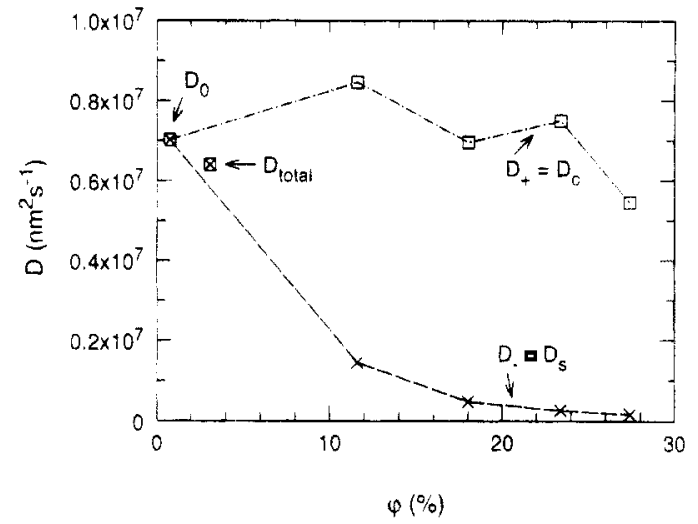

Figure 8. Diffusion coefficient of the rod mode(s) at $k=$ $0.0213 \mathrm{~nm}^{-1}$ as a function of the volume fraction.

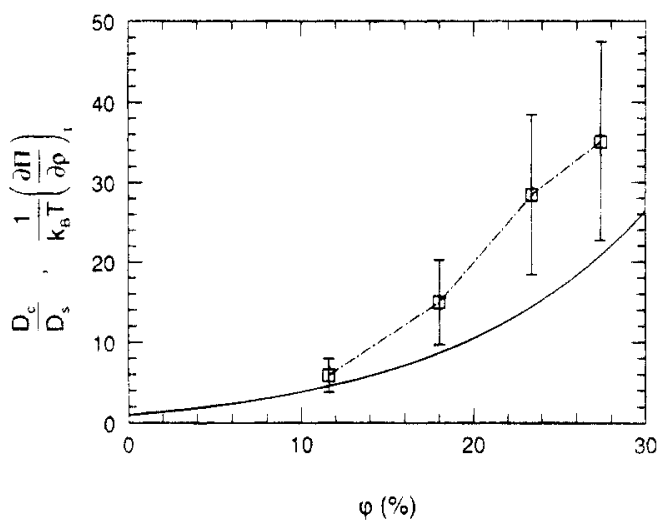

Figure 9. Measured $D_{\mathrm{d}} D_{\mathrm{s}}(\square)$ compared to the calculated $1 / k_{\mathrm{B}} T(\partial \Gamma \mathrm{\partial} \partial)_{\mathrm{T}}(-)$ using eq 11 plotted as a function of the volume fraction.

noted. A similar decrease has been noted before in experiments on rigid polymers. ${ }^{7,40}$ Its origin is unclear.

Disregarding possible polydispersity effects, the collective diffusion coefficient can be expressed as

$$
D_{\mathrm{c}}=\frac{(\partial \Pi / \partial \varrho)_{\mathrm{T}}}{f_{\mathrm{c}}}
$$

with $f_{c}$ the friction coefficient for the collective movement of rods, which is equal to the friction coefficient for sedimentation. ${ }^{41}$ As our measured collective diffusion coefficient is (almost) independent of the concentration, eq 26 demands that the increase in $(\partial \Pi / \partial \varrho)_{\mathrm{T}}$ must be balanced by the increase in $f_{c}$. For the self-diffusion coefficient, we can write

$$
D_{\mathrm{s}}=\frac{k_{\mathrm{B}} T}{f_{\mathrm{s}}}
$$

where $f_{\mathrm{s}}$ is the friction coefficient for single rods. For a dilute dispersion, $(\partial \Pi / \partial \varrho)_{\mathrm{T}}=k_{\mathrm{B}} T$ and $f_{\mathrm{c}}=f_{\mathrm{s}}$, so that $D_{\mathrm{c}}$ $=D_{\mathrm{s}}=D_{0}$. Although $f_{\mathrm{c}}$ and $f_{\mathrm{s}}$ are fundamentally different, some indications have been found that $f_{\mathrm{c}}$ and $f_{\mathrm{s}}$ are approximately equal. ${ }^{40}$ If this is also true for our rods, then $D_{\mathrm{c}} D_{\mathrm{s}}$ should be equal to $1 / k_{\mathrm{B}} T(\partial \Pi / \partial \rho)_{\mathrm{T}}$. Making this comparison in Figure 9 , where $1 / k_{\mathrm{B}} T(\partial \Pi /$ $\partial Q)_{T}$ is calculated from eq 11 , shows that this is not the case. This indicates that $f_{\mathrm{c}}$ and $f_{\mathrm{s}}$ are not quantitatively the same, although we note that the calculated $1 / k_{\mathrm{B}} T(\partial \Gamma /$ $\partial o)_{\mathrm{T}}$ is not rigorously correct.

A single-exponential plot of $D_{0} / D_{s}$ is linear in the entire concentration range as is shown in Figure 10. A double-logarithmic plot of our $D_{\mathrm{s}}$ against $\varphi$, however, gives a linearly plot for the four highest concentrations, yielding a slope of -2.54 . As far as we know, only one 


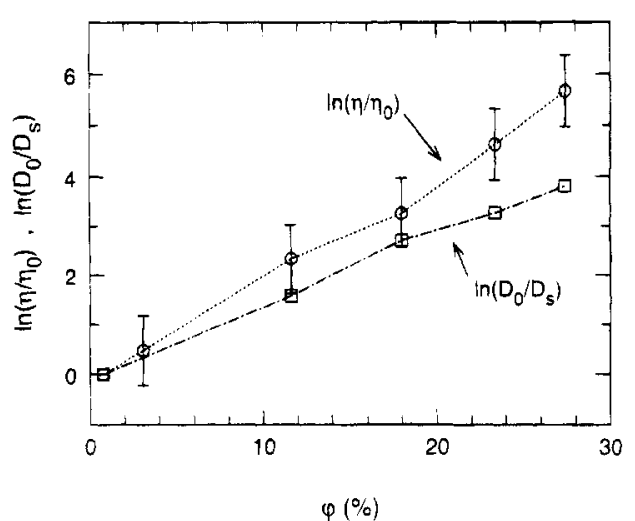

Figure 10. Logarithmic plot of the exchange diffusion and the low-shear viscosity data as a function of the volume fraction. The relative viscosities are obtained from the cluster diffusion coefficient (see section 6.2), giving rise to large errors due to the correction for the estimated increase in cluster size.

experimental study on the self-diffusion of rodlike particles has been reported in the literature. ${ }^{33}$ The study concerns forced Rayleigh scattering measurements on solutions of a 150-base pair DNA fragment far below the concentration of the isotropic nematic phase separation. The self-diffusion coefficient decreases with concentration, and a double-logarithmic plot of the self-diffusion constant against the concentration gives a limiting slope of about -0.5 . The much higher slope of -2.54 found in our measurements can be attributed to the different concentration range.

The diffusion coefficient of the clusters decreases strongly with concentration (Table 3 ). If the dispersion of rods is regarded as a dispersion medium for the clusters, this strongly decreasing diffusion coefficient can be attributed to an increase in viscosity of the rod dispersion in combination with an increase in cluster size. Consequently, correcting the diffusion coefficient of the clusters for the increase in cluster size yields the low-shear viscosity $(\eta)$ of the dispersion,

$$
\frac{\eta}{\eta_{0}}=\frac{D_{3}\left(\varphi_{1}\right) R_{\mathrm{g}}\left(\varphi_{1}\right)}{D_{3}(\varphi) R_{\mathrm{g}}(\varphi)}
$$

with $\eta_{0}$ the viscosity of the solvent, $D_{3}$ the diffusion coefficient of the clusters as given in Table $3, \varphi_{1}$ the lowest volume fraction $(0.755 \mathrm{v} / \mathrm{v} \%)$, and $R_{\mathrm{g}}$ the Guinier radius of the clusters (see section 5 ). Unfortunately, we only have a rough estimate of $R_{\mathrm{g}}\left(\varphi_{1}\right) / R_{\mathrm{g}}(\varphi)$, giving viscosities that are estimated to be accurate within a factor of 2 .

These viscosities are compared to the exchange (or "self-") diffusion coefficients, following the results for sterically stabilized (uncharged) silica spheres, where the long-time self-diffusion coefficient was found to be almost inversely proportional to the viscosity of the dispersion itself. ${ }^{37}$ Figure 10 gives this comparison for our grafted boehmite rods, showing the logarithm of the low-shear viscosity as well as the exchange ("average" self-) diffusion plotted against the volume fraction. The results indicate that the viscosity increases more strongly with concentration than the inverse of the exchange diffusion coefficient. This could be a result of the lengthwise diffusion of the rods, which is probably less hindered than the sidewise diffusion, or more or less equivalently the diffusion of larger species, such as clusters. However, the quantitative comparison with long-time self-diffusion results may be somewhat disturbed because our measurements are not completely in the small wave vector limit.

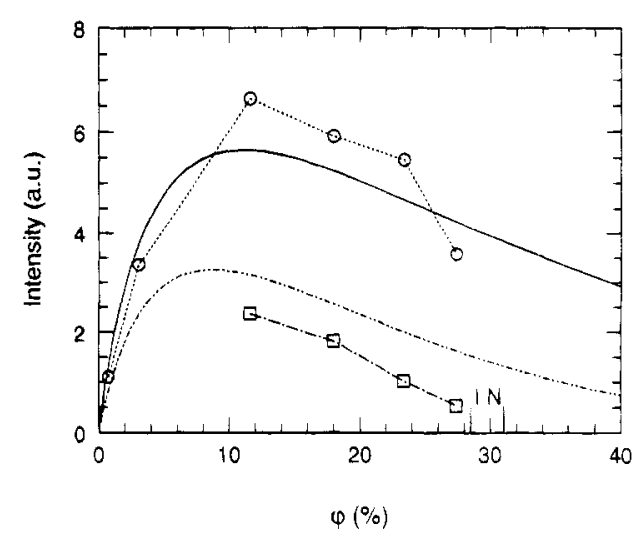

Figure 11. Scattered intensity of the rods as a function of the volume fraction compared to the SPT result. The symbols denote the measurements, which are connected to guide the eye. The upper curve $(O)$ gives the total rod intensity (mode $1+2$ ), and the lower curve ( $\square$ ) gives the scattering associated with osmotic compressibility fluctuations. The corresponding smooth curves give the SPT result. The concentrations of the isotropic nematic phase separation are denoted by I and $\mathrm{N}$.

Our viscosity results can be compared to tracer diffusion results as measured by dynamic light scattering on sphere diffusion in solutions of poly $(\gamma$-benzyl- $\alpha, L-$ glutamate) rods in dimethylformamide. ${ }^{42}$ The spheres had a radius of $60.4 \mathrm{~nm}$, and the poly $(\gamma$-benzyl- $\alpha, \mathrm{L}-$ glutamate) rods had a length of $70 \mathrm{~nm}$. With this system, no significant difference could be observed between a direct measurement of the viscosity and the viscosities obtained from the tracer diffusion, confirming our method of measuring the viscosity using the cluster diffusion. The viscosity results in ref 42 yield $\eta / \eta_{0}=$ $\exp \left(\alpha c^{\nu}\right)$, with $v=0.84$. Our data yields $v=1.0$, as is demonstrated in Figure 10.

Mode Amplitudes. The relative mode amplitudes in Table 3 are now used to calculate the absolute scattered intensities of the rods, which are obtained by multiplication of the relative mode amplitude with the overall scattered intensity as obtained from static light scattering. Mode 1 gives the scattered intensity due to the overall concentration fluctuation, and the sum of modes 1 and 2 gives the total scattered intensity of the rods. These intensities are compared to theoretical results from the SPT (eqs 21-24, using the EM dimensions with eq A9) in Figure 11. It should be noted that there is one common scaling factor for the intensity of both theoretical curves, i.e., their relative intensities are fixed by the SPT.

We see that there is semiquantitative agreement between theory and experiment. The trends of the theory and the experimental results correspond quite well. The intensity maximum is found at approximately the predicted volume fraction, and the intensity of the collective mode decreases relative to the exchange mode for higher volume fractions. This behavior is similar to that observed for spheres. ${ }^{6,13,22}$

The fact that there is only semiquantitative agreement does not necessarily mean that the SPT is in error, as the particles are not the ideal polydisperse spherocylinders as assumed in the SPT calculations. The most important disagreement between theory and experiment is that the total scattered intensity relative to the collective mode intensity is higher in the experiment than in the theory. This deviation may be due to the neglect of the anisotropy of the refractive index as well as to the polydispersity in the average refractive index (i.e., the average of the boehmite and steric stabilization layer). 


\section{Conclusions}

We performed static and dynamic light-scattering experiments at small scattering vectors on dispersions of rods with a length over width ratio of 6.4 , up to concentrations close to the isotropic nematic phase separation. The combination of static and dynamic light scattering and electron microscopy allowed for the demonstration of a moderate degree of clustering as well as for the separation of the scattered intensity in part due to the free rods and in part due to the clusters.

The main feature of the dynamic light scattering of the rods part is the separation in a collective diffusion mode and an exchange (or self-) diffusion mode, which is attributed to the polydispersity of the particles. The diffusion coefficient associated with the collective mode is (almost) independent of the concentration, while the diffusion coefficient corresponding to the exchange diffusion mode decreases exponentially with the concentration. The corresponding mode amplitudes are in semiquantitative agreement with the scaled particle theory (Figure 11). The trends of the behavior of the diffusion coefficients and mode amplitudes are similar to those observed for polydisperse spherical particles. To perform more accurate measurements regarding diffusion coefficients, mode amplitudes, and possible additional modes that we may have missed, $7,9,18,24,29$ a strong reduction of the degree of clustering will probably be necessary.

An indirect estimate of the low-shear viscosity of our dispersions is obtained from the cluster diffusion coefficient and shows an exponential concentration dependence, which is rather close to the weakly stretched exponential concentration dependence obtained with solutions of a rigid polymer. ${ }^{42}$ The inverse viscosity obtained here appears to decrease more strongly with concentration than the exchange diffusion coefficient.

We note, as a point of general interest, that for measurements at small scattering vectors, and arbitrary shaped particles, the effect of polydispersity is always expected to give an additional mode due to the exchange of different species from the polydisperse distribution, in addition to a polydisperse distribution of the measured modes. This should also apply to rigid polymers, for which an exchange mode has not been noticed so far. However, in recent dynamic light-scattering experiments on the semiflexible polymer ( $p$ oly $(\gamma$-benzyl$\alpha, \mathrm{L}$-glutamate), Delong and Russo $0^{40}$ gave an accurate description of a slow mode that only occurred at high concentrations and the origin of which remained un. clear. Following our results, this mode might be an exchange mode, as the polymer was shown to be somewhat polydisperse. Furthermore, it is noted that the SPT predicts a much smaller exchange mode amplitude for rods with only length polydispersity, as compared to rods with similar width polydispersity. ${ }^{14}$ This may explain why an exchange mode has remained unnoticed in studies on rigid polymers.

Acknowledgment. This work was supported by the Netherlands Foundation for Chemical Research (SON) with financial aid from the Netherlands Organization for Scientific Research (NWO).

\section{Appendix. Description of Length and Width Distribution}

Theoretical model calculations including polydispersity are carried out for the form factor, the autocorrelation functions, and the scattered intensity according to the SPT. These calculations are performed on the basis of the average dimensions obtained from electron microscopy, using a length and width distribution function. Here, we use a bivariate log-normal distribution, $f(L, D)$, because it captures at least two important features of the distributions obtained from electron microscopy, namely that they are skewed and that $L$ and $D$ are correlated. The bivariate log-normal distribution is a bivariate normal distribution ${ }^{43}$ of the logarithmic variables $l=\ln (L)$ and $d=\ln (D)$ and is defined by

$$
\begin{aligned}
& f(L, D)=\frac{1}{2 L D \sqrt{z}} \times \\
& \exp \left[-\frac{(l-\bar{l})^{2} \sigma_{d}^{2}-(l-\bar{l})(d-\bar{d}) \operatorname{cov}(l, d)+(d-\bar{d})^{2} \sigma_{1}^{2}}{2 z}\right]
\end{aligned}
$$

where

$$
z=\sigma_{1}^{2} \sigma_{\mathrm{d}}^{2}-[\operatorname{cov}(l, d)]^{2}
$$

Here, $\sigma$ is the standard deviation which, for example, for the diameter $D$ is defined by

$$
\sigma_{\mathrm{D}}^{2}=\overline{(D-\bar{D})^{2}}=\overline{D^{2}}-\overline{\mathrm{D}}^{2}
$$

and the covariance of $l$ and $d$ is given by

$$
\operatorname{cov}(l, d)=\overline{(l-\bar{l})(d-\bar{d})}=\overline{l d}-\vec{l} \bar{d}
$$

The geometric (logarithmic) standard deviations and averages for $l$ and $d$ can be calculated from their equivalents in terms of $L$ and $D$. For example, for the diameter we have

$$
\sigma_{\mathrm{d}}^{2}=\ln \left[\frac{\sigma_{\mathrm{D}}^{2}}{\bar{D}^{2}}+1\right]
$$

and

$$
\bar{d}=\ln \left[\bar{D} \exp \left[-(1 / 2) \sigma_{\mathrm{d}}^{2}\right]\right]
$$

The geometric covariance is given by

$$
\operatorname{cov}(l, d)=\ln \left[\frac{\operatorname{cov}(L, D)}{\bar{L} \bar{D}}+1\right]
$$

We express the covariance as the dimensionless correlation coefficient

$$
r(L, D)=\frac{\operatorname{cov}(L, D)}{\sigma_{\mathrm{L}} \sigma_{\mathrm{D}}}
$$

A double integration using the bivariate log-normal distribution of eq A1 yields for arbitrary positive integers $a$ and $b$

$$
\begin{aligned}
& \overline{L^{\mathrm{a}} D^{\mathrm{b}}}= \\
& \bar{L}^{\mathrm{a}} \bar{D}^{\mathrm{b}}\left[\frac{\sigma_{\mathrm{D}}^{2}}{\bar{D}^{2}}+1\right]^{1 / 2 b(b-1)}\left[\frac{\sigma_{\mathrm{L}}^{2}}{\bar{L}^{2}}+1\right]^{1 / 2 a(a-1)}\left[\frac{\operatorname{cov}(L, D)}{\bar{L} \bar{D}}+1\right]^{a b}
\end{aligned}
$$


in eq 22 , from which the scattered intensity within the SPT is calculated.

\section{References and Notes}

(1) Hansen, J. P., Levesque, D., Zinn-Justin, J., Eds. Liquids, freezing and the glass transition, Les Houches Session LI, part $I$ and $I I$; North-Holland: Amsterdam, 1991.

(2) Pusey, P. N.; Tough, R. J. A. In Dynamic Light Scattering, Applications of Photon Correlation Spectroscopy; Pecora, R., Ed.; Plenum Press: New York, and London, 1985; pp 85179.

(3) Kerker, M. The scattering of light and other electromagnetic radiation; Academic Press: New York, 1969.

(4) Huglin, M. B. Light scattering from polymer solutions; Academic Press: London, 1972

(5) Chu, B. Laser Light Scattering, Basic Principles and Practice, 2nd ed.; Academic Press: London, 1991; 17.

(6) Agterof, W. G. M.; Zomeren, J. A. J. van; Vrij, A. Chem. Phys. Lett. 1976, 43, 363-367.

(7) Russo, P. S. Dynamic light scattering from rigid and nearly rigid rods. In Dynamic light scattering, The method and some applications; Brown, W., Ed.; Clarendon Press: Oxford, 1993.

(8) Hagenbüchle, M.; et al. Physica A 1990, 169, 29-41. Graf, C.; et al. J. Chem. Phys. 1991, 95, 6284-6289. Maier, E. E.; Fraden, S.; et al. Macromolecules 1992, 25, 1125-1133.

(9) Tracy, M. A.; Pecora, R. Annu. Rev. Phys. Chem. 1992, 43, $525-557$.

(10) Buining, P.; Pathmamanoharan, C.; Jansen, J. B. H.; Lekkerkerker, H. N. W. J. Am. Ceram. Soc. 1991, 74, 1303-1307.

(11) Buining, P. A.; Veldhuizen, Y. S. J.; Pathmamanoharan, C. Lekkerkerker, H. N. W. Colloids Surf. 1992, 64, 47-55.

(12) Pusey, P. N.; Fijnaut, H. M.; Vrij, A. J. Chem. Phys. 1982, $77,4270-4281$

(13) Veluwen, A. van; Lekkerkerker, H. N. W.; Kruif, C. G. de; Vrij, A. J. Chem. Phys. 1988, 89, 2810-2815.

(14) Bolhuis, P. G.: Lekkerkerker, H. N. W. Physica A 1993, 196, $375-388$.

(15) Buitenhuis, J.; Dhont, J. K. G.; Lekkerkerker, H. N. W. J. Colloid Interface Sci. 1993, 162, 19-24.

(16) Shimada, T.; Doi, M.; Okano, K. J. Chem. Phys. 1988, 88, $2815-2821$

(17) Doi, M.; Shimada, T.; Okano, K. J. Chem. Phys. 1988, 88, $4070-4075$.

(18) Shimada, T.; Doi, M.; Okano, K. J. Chem. Phys. 1988, 88, 7181-7186.
(19) Maeda, T. Macromolecules 1989, 22, 1881-1890.

(20) Schoot, P. van der; Odijk, T. Macromolecules 1990, 23, 41814182.

(21) Schoot, P. van der. Macromolecules 1992, 25, 2923-2927.

(22) Vrij, A. J. Colloid Interface Sci. 1982, 90, 110-116.

(23) Hallett, F. R.; Nickel, B.; Craig, T. Biopolymers 1985, 24 , 947-960.

(24) Zero, K. M.; Pecora, R. Macromolecules 1992, 15, 87-93.

(25) Perutz, M. F. Acta Crystallogr. 1953, 6, 859-864. Bragg, W. L.; Pippard, A. B. Acta Crystallogr. 1953, 6, 865-867.

(26) Aragón, S. R.; Pecora, R. J. Chem. Phys. 1985, 82, 53465353.

(27) Broersma, S. J. Chem. Phys. 1960, 32, 1632-1635.

(28) Tirado, M. M.; García de la Torre, J. J. Chem. Phys. 1979, $71,2581-2587$.

(29) Doi, M.; Edwards, S. F. J. Chem. Soc., Faraday Trans. 21978 , 74,560 .

(30) Doi, M.; Edwards, S. F. The Theory of Polymer Dynamics; Clarendon Press: Oxford, 1986.

(31) Marrucci, G.; Grizzuti, N. J. Polym. Sci., Polym. Lett. Ed. $1983,21,83-86$.

(32) Marrucci, G.; Grizzuti, N. J. Non-Newtonian Fluid Mech. 1984, 14, 103-119.

(33) Wang, L.; Garner, M. M.; Yu, H. Macromolecules 1991, 24, 2368-2376.

(34) Rosenfeld, Y. J. Chem. Phys. 1988, 89, 4272-4287.

(35) Buitenhuis, J.; Philipse, A. P.; Dhont, J. K. G.; Lekkerkerker, H. N. W. Prog. Colloid Polym. Sci. 1993, 93, 188.

(36) Weast, R. C. Handbook of Chemistry and Physics, 62nd ed.; CRC Press, Inc.: Boca Raton, FL, 1981-1982

(37) Blaaderen, A. van; Peetermans, J.; Maret, G.; Dhont, J. K. G. J. Chem. Phys. 1992, 96, 4591-4603.

(38) Imhof, A.; Blaaderen, A. van; Maret, G.; Mellema, J.; Dhont, J. K. G. J. Chem. Phys. 1994, 100, 2170-2181.

(39) Kops-Werkhoven, M. M.; Fijnaut, H. M. J. Chem. Phys. 1982, $77,2242-2253$.

(40) DeLong, L. M.; Russo, P. S. Macromolecules 1991, 24, 61396155.

(41) Kops-Werkhoven, M. M.; Vrij, A.; Lekkerkerker, H. N. W. J. Chem. Phys. 1983, 78, 2760-2763.

(42) Tracy, M. A.; Pecora, R. Macromolecules 1992, 25, 337-349.

(43) Hogg, R. V.; Craig, A. T. Introduction to mathematical statistics, 4th ed.; Collier Macmillan: New York and London, 1970; p 405 . 